\title{
A $360^{\circ}$ Vision for Virtual Organizations Characterization and Modelling: Two Intentional Level Aspects
}

\author{
Luz-María Priego-Roche, Dominique Rieu, and Agnès Front \\ LIG Laboratory, SIGMA Team, BP 72, 38402 Saint Martin d'Héres, Cedex France \\ \{Luz-Maria.Priego-Roche, Dominique.Rieu, Agnes. Front\}@imag.fr
}

\begin{abstract}
Nowadays, organizations aiming to be successful in an increasingly competitive market tend to group together into virtual organizations. Designing the information system (IS) of such virtual organizations on the basis of the IS of those participating is a real challenge. The IS of a virtual organization plays an important role in the collaboration and cooperation of the participants organizations and in reaching the common goal. This article proposes criteria allowing virtual organizations to be identified and classified at an intentional level, as well as the information necessary for designing the organizations' IS. Instantiation of criteria for a specific virtual organization and its participants, will allow simple graphical models to be generated in a modelling tool. The models will be used as bases for the IS design at organizational and operational levels. The approach is illustrated by the example of the virtual organization UGRT (a regional stockbreeders union in Tabasco, Mexico).
\end{abstract}

\section{Introduction}

Nowadays most organizations are subject to many events which affect their working methods: new competitors, new customer requirements, new technologies, etc. Globalization has accelerated these changes; consequently, companies seek new strategies to survive. Moreover, organizations do not work alone; they are conscious that they are no longer isolated entities and that they must have collaborate with other organizations in various ways in this changing environment. One way to deal with ever changing business opportunities is to form a Virtual Organization (VO). According to several authors [15] [4] 27] 38] 50, a VO could be considered as "An alliance for integrating competences and resources from several independent real companies, that are geographically dispersed. This integration is possible throughout the layout of an information systems infrastructure to satisfy customer's requirements, or to seize a business opportunity without having to form a new legal entity".

The organizational unit concept has changed through time starting from individual and group based structures 35, passing by organizational based functional departments [4], evolving to virtual organizations [15] [1] and virtual organization networks.

C. Godart et al. (Eds.): I3E 2009, IFIP AICT 305, pp. 427 442, 2009.

(C) IFIP International Federation for Information Processing 2009 
Many researchers agree that information and communication technologies play a fundamental role in a VO [18, 12, 14, 32. Thus, information systems facilitate cooperation, communication and collaboration of the VO's members. They support sharing resources and new working modes while preserving their individual administrative structures.

Information Systems (IS) have a dominating influence in the organization's ability to adapt to these changes; a company's agility strongly depends on the agility on its IS [4]. To function, a VO requires integrated IS, allowing services, collaboration and cooperation among the Participant Organizations (PO).

VO's information system development involves methodological and technical problems. The identification and representation of requirements are difficult for a "traditional" organization. Such tasks require more effort for a VO due to the large number of organizations and the differing backgrounds and cultures of the people involved.

This article proposes a framework for analyzing a $\mathrm{VO}$ based on a $360^{\circ}$ vision: intra-organizational (PO's individual properties), inter-organizational (collective properties) and extra-organizational (environmental properties). It particularly covers a set of aspects for characterizing a VO and its $\mathrm{PO}$ at an intentional level where collaboration definition and common objectives are emphasized. The aspects are taken from previous research on business management [18] 24] 26] 16] 20] 10] [2] and are intended to support the modelling of a VO allowing various actors (shareholder, user, project manager, business analyst) to obtain all necessary knowledge to conceive collaborative information systems through a VO IS methodology. More precisely, this work aims to facilitate:

- setting up agreements among the different POs by identifying the set of properties linked to their alliance (identification of PO, compromises, objectives, etc.),

- formalizing requirements, a necessary step for defining organizational processes and consequently the adapted collaborative IS.

For doing so, we identify, classify and formalize [36] the intentional characteristics of the VO, to represent them in the form of simple graphic and textual models, and to provide a software platform to capture and manage the characteristics as much as the models. These two approaches have proved to be effective means in helping actors to understand and communicate that understanding among them [5] 43] 28] 17] in various scientific fields 34] 37]. Properties formalization is carried out with UML diagrams, an accepted standard for covering conceptual elements 3.

Our research method is strongly based on a case study, the Tabasco's Regional Stockbreeders Union's case study (UGRT) 1 , based on one of the authors (Priego) working experience in this organization. The UGRT gathers several companies working in the cattle industry. It offers multiple services and products to its members in a strong cooperation atmosphere in order to increase the economic revenue of cattle production. It is formed by several enterprises (a slaughter-house, a

\footnotetext{
${ }^{1}$ Union Ganadera Regional de Tabasco (http://www.ugrtab.com)
} 
packing facility, a retail store, etc) illustrated in Figure1. Among them, the stockbreeders are regarded as members of the organization and have the right to use the services offered by the companies, they are grouped in Local Stockbreeders Associations.

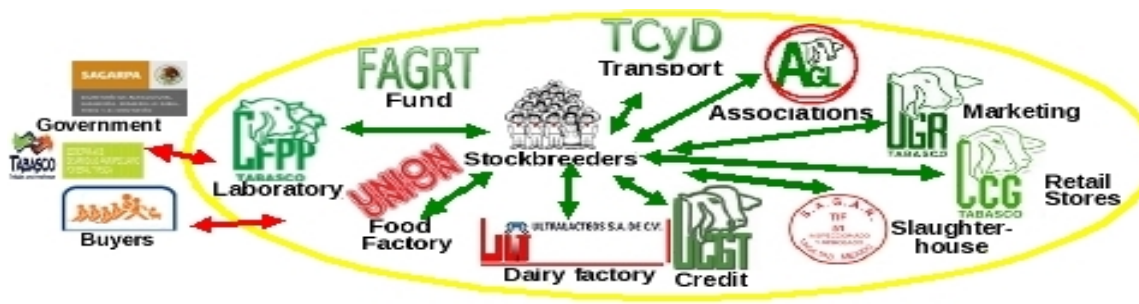

Fig. 1. Case study: the VO UGRT

Thereafter, we specify a $360^{\circ}$ vision for the VO (section 2). A description of the relevant aspects to study for a $\mathrm{VO}$ at the intentional level is presented in section 3. Two of these aspects are detailed in sections 4 and 5, each of them is broken down, formalized in a UML diagram and illustrated with a graphic model. The illustrations are based on our case study and on a prototype tool for managing the aspects and their representation in graphic models generated automatically from the seized data. Section [6] summarizes our proposals and prospects for future study.

\section{A $360^{\circ}$ Vision for a $\mathrm{VO}$}

Different points of view are needed to ease IS requirement elicitation of the VO. We propose to characterize the VO's requirements across three levels covering a $360^{\circ}$ vision that allow us to analyse the $\mathrm{VO}$ from different angles and complement other visions [33] 13] (Figure 2):

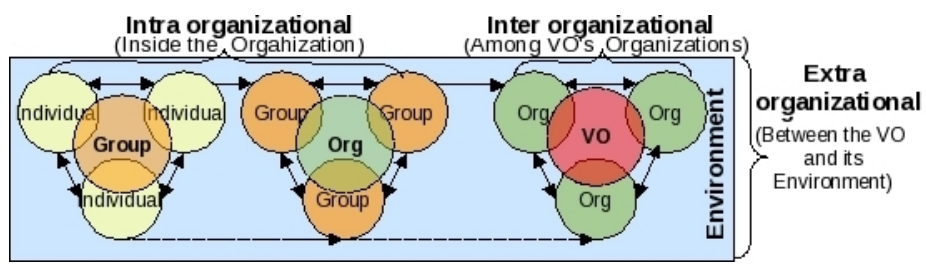

Fig. 2. Intra, Inter and Extra Organizational levels

- Intra-organizational level: it has an internal orientation focusing on the relationships among the functional actors (individuals and groups) of the participant organizations (POs) of the VO. This level involves the traditional structure of an organization. 
- Inter-organizational level: it focuses on the relationships among the POs forming the VO. This level involves a new collaborative strategy among several organizations.

- Extra-organizational level: it has an external orientation focusing on the relationship between the VO and its macro environment. This level involves VO's outside actors influences.

In addition, different levels of abstraction are also needed 40]:

- Intentional level, where collaboration definition and common objectives are emphasized.

- Organizational level, where formalization of the business processes is carried out.

- Operational level, where the actions for executing the proposed business processes are detailled, describing the structure and the operation of the IS.

Figure 3 represents the proposed $360^{\circ}$ vision that combines the extra, inter and intra organizational points of view and the intentional, organizational and operational abstraction levels. The work presented in this article focuses on the intentional level whose major aspects are described in the following section.

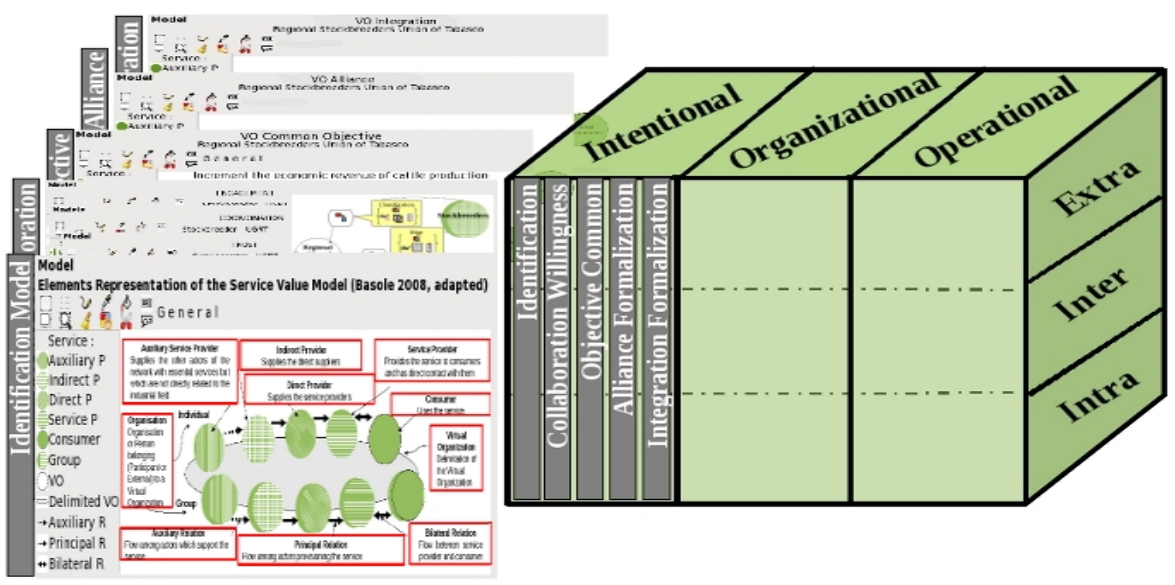

Fig. 3. $360^{\circ}$ vision of the Virtual Organization

\section{Intentional Level}

The intentional characterization is an essential starting point for organizational and operational modelling of a collaborative IS for a VO. Many research work have highlighted the properties that characterize a virtual organization. Some of these approaches are interested in the collaborative aspects $([26,[32])$, others emphasize business processes ([38], 47]), or identifying the common benefits [31, 
or the physical dispersion [1. Specific works dedicated to small and medium-size companies (SME Collaborate2, ECOLEAD 3 for example), offer methods and technological infrastructures dedicated to supporting collaboration among these companies. Several modelling languages dedicated to requirement engineering have been proposed to represent organizations: $i^{*}$ framework [21, KAOS [46], CREWS [30, MAP [8], e3 VALUE [19], Service Value Networks [37, etc. The charts used in this article are inspired by the Service Value Networks modelling language 37.

According to 23] a VO life-cycle is composed by identification, formation, operation/evolution and dissolution/termination, we suggest analyzing the VO according to five relevant aspects that support the formation phase of a VO through collaboration alliance 33] (Figure 4):

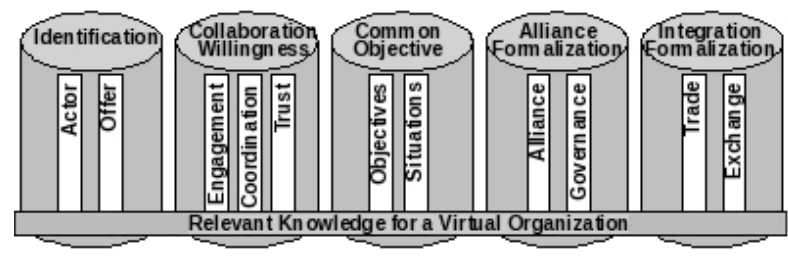

Fig. 4. Major aspects for studying a Virtual Organization

- Identification: it characterizes the VO and its composition in terms of organizations and offered services (described in detailed in section 4).

- Collaboration Willingness: it characterizes the compromises among the Participant Organizations (PO) to establish the alliance and to work together (described in detailed in section 5)

- Common Objective: it characterizes the shared goal and the directions to be followed for reaching it. The latter could answer customer's needs (integral services), satisfy companies' objectives (to share costs, benefits, to create more effective processes) [22, make new business (new markets, new products or services), confront difficulties (absence of knowledge). Usually, objectives emerge from situations that could be classified in opportunities (circumstances favoring the alliance) or problems (difficulties that justify exploring the alliance).

- Alliance Formalization: it characterizes the way the alliance is built, more often on agreements or contracts than on mergers and/or acquisitions. Dynamism of the VO is linked to the short or long term alliance established 33. Moreover, it defines the strenght each organization has in the VO: strong-weak (one leader PO defining the rules, procedures for achieving the objective) or equal (each PO has the same power for making decisions).

\footnotetext{
${ }^{2}$ Small and Medium Enterprise Collaborate, [http://www.smecollaborate.com/]

${ }^{3}$ European Collaborative Networked Organisations Leadership Initiative, [http:// ecolead.vtt.fi/]
} 
- Integration Formalization: it characterizes the elements the organizations agree to share as part of their competencies and their resources. It defines the POs business processes offered to the VO and their impact in the service value generation. Each PO determines the resources willing to give and expecting to receive from the alliance, it requires defining the mechanisms to ease the electronic integration patterns: information (data, text, messages, images, voice) and communication (connection, access, transfer) [10] [50].

To define the VO more precisely, we propose to identify from each aspect, a set of sub-aspects, criteria and sub-criteria (Figure 5). Some of these criteria relate to the VO in general (generic criterion), other criteria are specific to the PO.

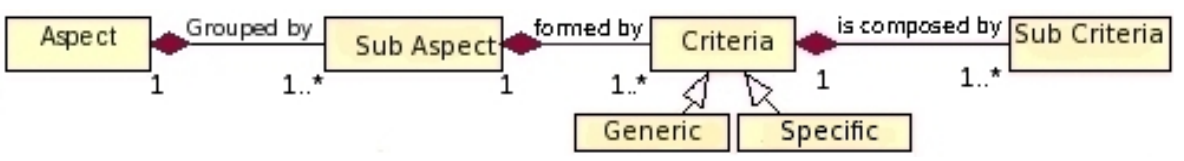

Fig. 5. Aspects decomposition

Each aspect (or one of its sub-aspects) is formalized by a class diagram (example Figure 6). A criterion is represented by a stereotyped class. Sub-criteria are represented either by simple attributes, or by associations linking the criterion class to the value class, describing the sub-criterion possible values. An instance example of these models, resulting from our case study, is represented in the form of prototype screens which are in process of development (example Figure 7). From this instantiation it is possible to deduce automatically simple graphic models (example Figure 8). The next two sections detail the Identification and Collaboration Willingness aspects.

\section{Identification Aspect}

The Identification Aspect is composed of two sub-aspects detailed below and formalized in Figure 6 .

- The Actor's goal is to identify and characterize the organizations (independently of their participation in the VO) as well as the VO. It is composed of two criteria.

- Organizations. Each organization is characterized by a name, a description, a size, a location (city, state, country), a constitution (individual organization or group) and an activity sector. The size is given based on a scale from Small to Large, while taking into account the number of employees, the turnover, the market share and the organization peculiarities. The peculiarities describe common properties to consider (e.g. "Manual Work for small stockbreeders"). An individual organization is not composed of other organizations. A group organization is a subsidiary of a 
holding organization which controls or guides the groups' activities. Activity sectors (specific and general) characterize the organization in its business context; we use those proposed by NAICS4. The sectors are characterized by a code, a name and a description; they are related to activities (example of specific activity: Livestock production; examples of general activities: Agriculture, Forestry and Fishing).

- Virtual Organization. A VO has a name, a description, a creation date and a specific activity sector. The VO is composed of organizations (at least two), each of whom plays a role in the VO: either as a Participant Organization (PO) in the alliance or as an External Organization (EO); the latter is not part of the alliance but it interacts directly with the VO either soliciting or rendering services. The roles have fixed durations set by starting and termination dates.

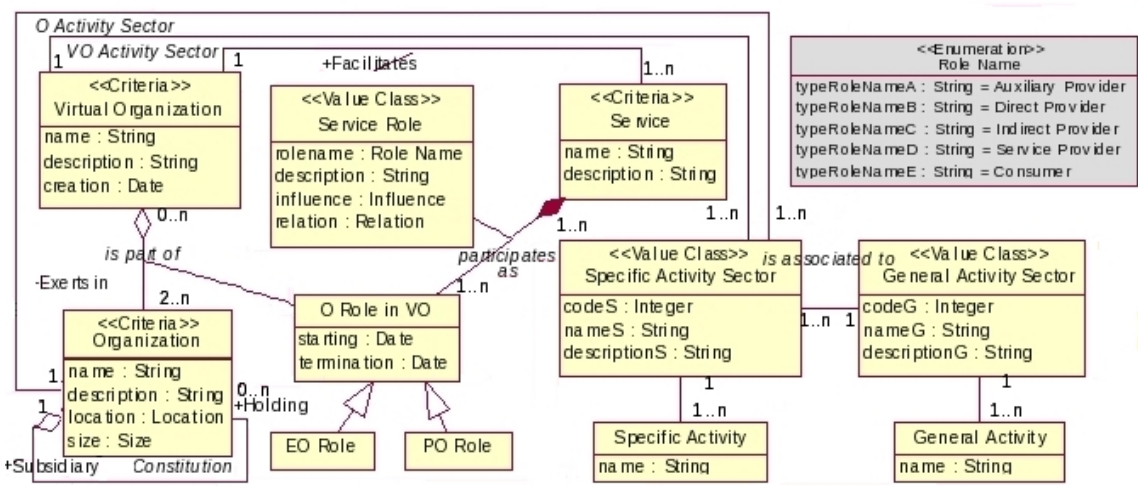

Fig. 6. Identification model

- The Offer's aim is to identify and characterize the services offered by the organizations and the roles the organizations play to produce these services. It is composed of two criteria.

- Services. A VO provides one or more services without making a distinction between a physical product (like the meat for example) or a service (like the distribution of the meat) 41. A Service has a name and a description.

- Role. Each PO or EO has a role in the achievement or the consumption of the service. It can be a role of provisioning or using the service (this means the relationship of the PO or the EO to the service). This role can be essential or secondary for the achievement of the service, meaning the influence on the activity by the PO or EO. The identified roles come from the work of 37:

\footnotetext{
${ }^{4}$ North American Industry Classification System, [http://www.census.gov/epcd/naics02/naicod02.htm]
} 
* an auxiliary service provider supplies the other actors of the network with essential services but which are not directly related to the industrial field,

* an indirect provider supplies the direct suppliers,

* a direct provider supplies the service providers,

* a service provider provides the service to consumers and has direct contact with them,

* a consumer uses the service.

Figure 7 illustrates an instance of this aspect. The top left screen allows users to assign organizations to a VO (here the Regional Stockbreeders Union of Tabasco), either as a PO or an EO. The top right screen characterizes each organization (size, location, etc.), for example Stockbreeder, and the bottom right screen lets users associate its activity sectors. Finally, the VO service is shown in the lower left screen and the Stockbreeder's role in the service is shown in the overlapped window on the bottom right.

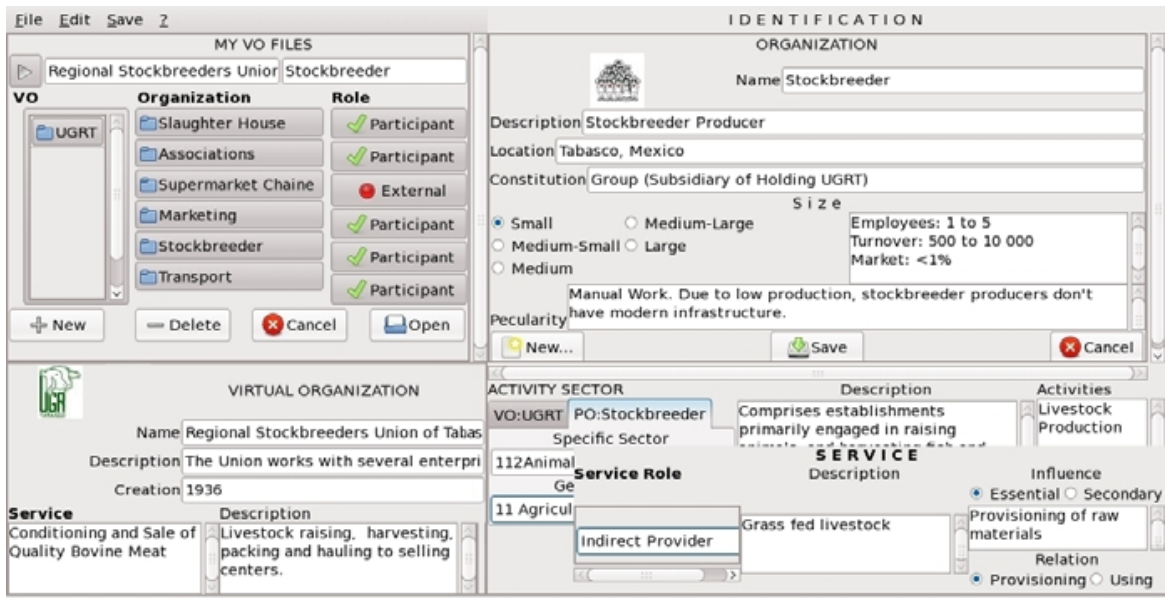

Fig. 7. Identification instance

From these properties, graphic representations can be generated in the form of models. For example, Figure 8 shows the set of organizations which form the VO of our case study based on an adaptation of [37]'s modelling language: the POs (Stockbreeder, Associations, Slaughter-house, Transport, Marketing) and the EOs (Supermarket Chains, Government Regulators, Meat Consumers) which compose the service "Conditioning and Sale of Quality Bovine Meat ". The roles of each organization or organization groups and their relationships are also illustrated. 


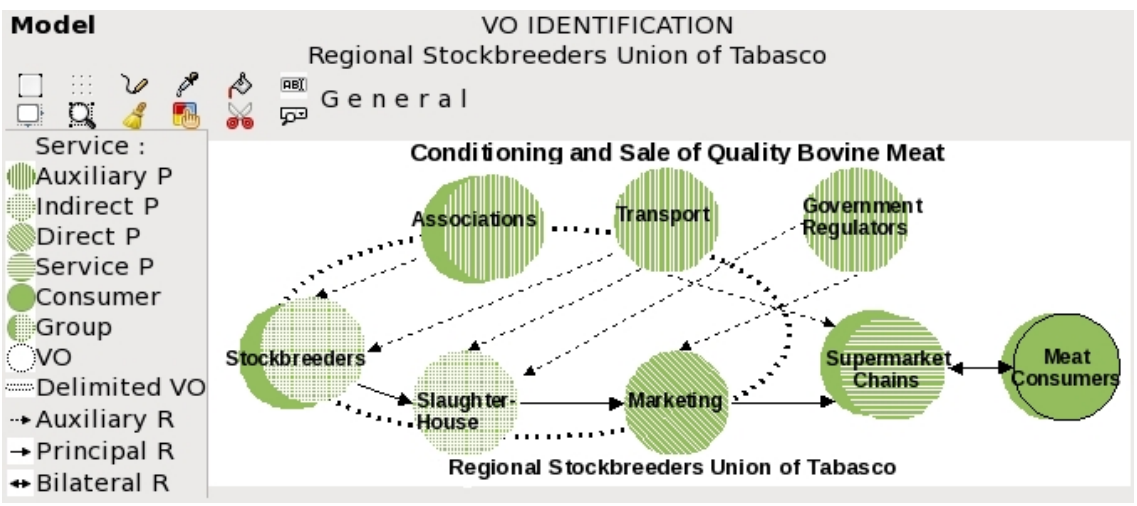

Fig. 8. Graphic representation of the Regional Stockbreeders Union VO

\section{Collaboration Willingness Aspect}

The Collaboration Willingness aspect is composed of three sub-aspects detailed below:

- Engagement qualifies the availabilities and the investments which the participant organizations are ready to engage (see Figure 91).

- The availability that the PO is ready to give is described by a time reserved for the relationship, a priority the PO assigns to this project compared to other projects and the PO's adaptability to changes. All these attributes can have a value estimated by a scale from Low, MediumLow, Medium, Medium-High, to High.

- The investments concern the elements that each PO devotes to the VO, for example financial or material assets, human, relational or organizational capital. This typology is suggested by [20. An investment thus has a type (for example financial credit), a frequency which can be constant, sporadic or event-triggered, and an impact (direct or indirect). Investments are described by sentences such as Give a contribution for each slaughtered cattle and by measurement objects (calculations and constraints).

In Figure 9, the availability criterion is represented in UML, the class "PO Role" has three attributes: time, priority and adaptability. We point out that the class "PO Role" is an association class characterizing the role of a PO in a VO (cf Figure 6]"PO Role" sub class of "O Role in VO"). Each instance of this class is related to investments which are related to measurements.

Figure 10 illustrates an instance of the Engagement Model for the PO "Stockbreeder" in terms of availability (top left screen), investment (in this case a financial type in the form of a "contribution evaluated by average costs"). This contribution is event-triggered (when the cattle is slaughtered) and has a direct 


\begin{tabular}{|c|c|c|c|c|}
\hline $\begin{array}{l}\text { «Enumeratio n»s } \\
\text { Scale }\end{array}$ & $1 \longdiv { 1 . . n }$ & $\begin{array}{l}<\varangle \text { Criteria }>> \\
\text { Inve stme nt }\end{array}$ & Callulate $\mathrm{M}$ & $\begin{array}{l}\text { Criteria } \gg \\
\text { a surem ent }\end{array}$ \\
\hline $\begin{array}{l}\text { typeScaleA }: \text { St ring = Low } \\
\text { typeScaleB }: \text { St ring = -Medium }\end{array}$ & O Ro le & $\begin{array}{l}\text { type : Type } \\
\text { description : String }\end{array}$ & $\begin{array}{l}1 \text { 1..n fo m } \\
\text { Constraint }\end{array}$ & ule : String \\
\hline 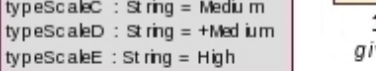 & $\begin{array}{c}1 . . \mathrm{n} \\
\text { ives }\end{array}$ & $\begin{array}{l}\text { frequency : Frequency } \\
\text { impact : Impact }\end{array}$ & $11 . . n$ & $\begin{array}{c}<<\text { Enumeration }>> \\
\text { Impact }\end{array}$ \\
\hline $\begin{array}{c}<\text { Ennmeration }>> \\
\text { Type }\end{array}$ & & & & $\begin{array}{l}\text { ty pe } \operatorname{lmpA}: \text { String }=\text { Direc } t \\
\text { typelmpB }: \text { String }=\text { Indirect }\end{array}$ \\
\hline $\begin{array}{l}\text { Typel rwA : String = Finantial Asset } \\
\text { Typel rw B : String = Material A sset }\end{array}$ & 1 & time: Scale & & $\begin{array}{l}<\text { Enumera tion }>> \\
\text { Freque ncy }\end{array}$ \\
\hline 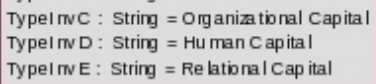 & & $\begin{array}{l}\text { priority: Scale } \\
\text { a daptability: Scale }\end{array}$ & $\begin{array}{l}\text { type Frequenc } \\
\text { type Frequenc } \\
\text { type Frequenc }\end{array}$ & $\begin{array}{l}\text { yA }: \text { String }=\text { Consta } n t \\
\text { yB }: \text { String }=\text { Spora dic } \\
\text { yC }: \text { String }=\text { Event-triggered }\end{array}$ \\
\hline
\end{tabular}

Fig. 9. Engagement model

impact on the VO. The bottom right screen shows a chart for the Engagement. The arcs bind the Stockbreeder PO with the VO; the squares delineated by continuous lines represent availabilities; the squares delineated by dotted lines do so for investments. For the latter, an icon inside the square illustrates its type.

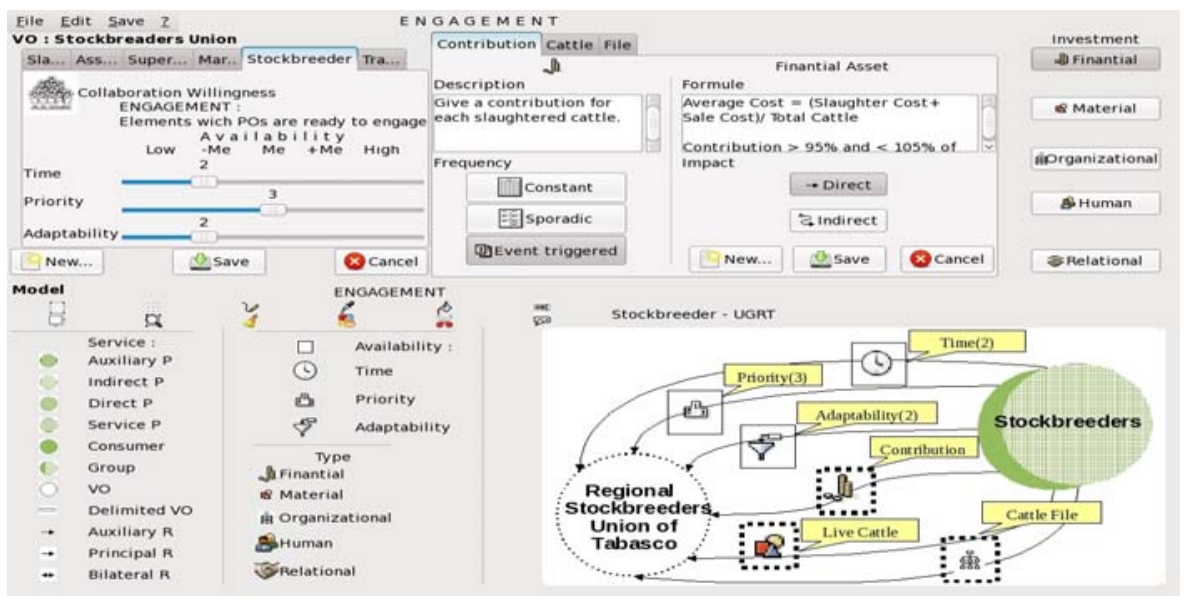

Fig. 10. Engagement instance

- Coordination characterizes the way the POs are organized to work together (see Figure 11). This is based on the work of [48, [15, 11], 49, to characterize inter-organizational coordination which is composed of the following two criteria:

- Elements involved that must be coordinated. Contrary to an investment, the coordinated elements remain the property of its organization. Each Element has a description and a dependence (coupled, uncoupled). An element can be a person or a process. The coordination of people 
requires knowing their profiles (preferences, personal information) and their work team (size, role). Process coordination implies specifying the task to carry out (planning, design) by characterizing its execution type (routine, non routine) and the information concerned (data, texts, messages, images, voice).

- Communication of each element is characterized based on CSCW5 29 and Denver 42 models. We describe communication through answering the questions where, when and how in terms of space, time and movement. Space includes a place (remote or local) and a state of presence (physical or virtual). Time includes a frequency (it can be constant, recurring, sporadic or event-triggered) and a moment (synchronous or asynchronous). Movement includes accessibility (mobile or fixed) and a direction (transmission, interaction, reception). This characterization determines the specific IS that offers the necessary functions of electronic communication to organize the interacting elements.

\begin{tabular}{|c|c|c|c|c|c|c|}
\hline \multirow{5}{*}{$\begin{array}{l}\text { Informa tion } \\
\text { typelnfA }: \text { String }=\text { Da ta } \\
\text { typelnfB }: \text { String }=\text { Te xt } \\
\text { typelnfC: String }=\text { Message } \\
\text { typelnfD }: \text { String }=\text { Image } \\
\text { typelnfE }: \text { String }=\text { Vo ice }\end{array}$} & \multirow{3}{*}{\multicolumn{2}{|c|}{\begin{tabular}{|l|}
\multicolumn{1}{|c|}{ Time } \\
frequency : Frequency \\
moment : Moment \\
\end{tabular}}} & & PO Role & & \multirow{2}{*}{$\begin{array}{c}<\ll \text { Crite ria }>> \\
\text { Comm unication }\end{array}$} \\
\hline & & & & 0. & & \\
\hline & & & & binat & & description : String \\
\hline & & nent & & & & where: Spa ce \\
\hline & $\begin{array}{l}\mathrm{a} \propto \\
\mathrm{d} \text { ie }\end{array}$ & $\begin{array}{l}\text { cee sb lity } \\
\text { ction }\end{array}$ & & $\begin{array}{l}<<\text { Criteri } \\
\text { Ele me }\end{array}$ & & $\begin{array}{l}\text { when : Time } \\
\text { how: Move ment }\end{array}$ \\
\hline Realization & & & & Sin & & \\
\hline \begin{tabular}{|l|} 
typeC oordA: String $=$ Routine \\
typeC oordB : String = Non Routine
\end{tabular} & \begin{tabular}{|l|} 
plac \\
pres
\end{tabular} & asence & & & & Process \\
\hline Dependence & & PeIs & & & task & String \\
\hline $\begin{array}{l}\text { typeDependenc eA : St ring = Couple } \\
\text { typeDependenc eB : St ring = U noou }\end{array}$ & & $\begin{array}{l}\text { profile: } \\
\text { team : } \$\end{array}$ & ring & & $\begin{array}{l}\text { re al } \\
\text { in fo }\end{array}$ & $\begin{array}{l}\text { zation: Realization } \\
\text { mation : Information }\end{array}$ \\
\hline
\end{tabular}

Fig. 11. Coordination model

Figure 11 proposes a coordination model where the criterion element is represented by a class. A PO can have one or more elements (people or process) to coordinate. Each element coordinated by a PO is characterized in terms of space, time and movement by an instance of the Communication class. Figure 12 illustrates an instance of the coordination model. The "Stockbreeder" has two elements to coordinate: a person type, manager and a process type, inventory (in top right screen). A graphic instance inspired from Basole's model is proposed in the bottom screen. The element types to coordinate are framed by pentagons, continuous sides for people and dotted sides for processes; they are attached to legends describing the attributes using icons.

- Trust among partners plays a decisive role in the alliance [6] 26] from group [39] to virtual organization perspective [7] 25]. In [9] Sauders et al conceptualizes it as Benevolence (acting in good will), Integrity (adherence to an accepted moral code), Competence (expectation of technically competent role

${ }^{5}$ Computer Supported Cooperative Work. 


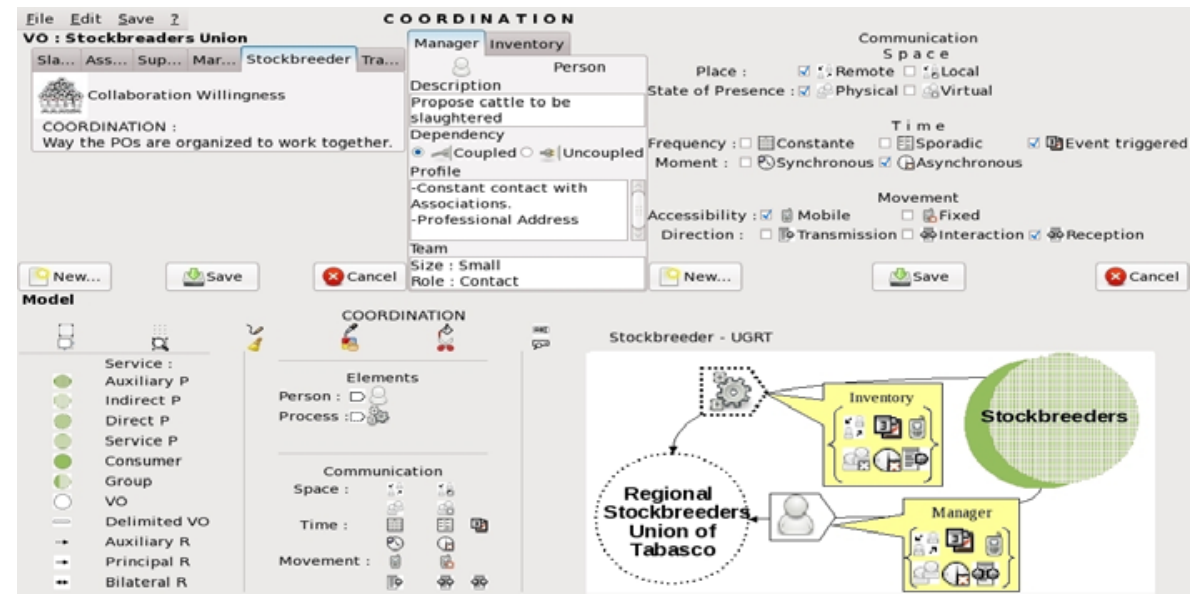

Fig. 12. Coordination instance

performance) and Predictability (acting in accordance to an expected behavior). We propose to describe it using the following criteria (see Figure 13):

- Functions are the control activities required to be vigilant and need to be regulated. A function carried out by a PO is characterized by a name, a description, an agreement among all PO on the importance of these functions, an achievement (optional or obligatory) and an execution. According to 16, the execution of these functions can be done either by giving entire freedom of action (indicating the independence of each PO to achieve its responsibilities), or by controlling them.

- Regulation of the functions ensures the good performance of the VO. A regulation is characterized by the type of element to control (for example active material) and its description (for example cattle), a visibility (if the PO supervises the execution of its proper functions, we talk about self-regulation; if it must debrief to other PO, we talk about surveillance), a control frequency (constant, sporadic, event-triggered) and penalization in case of violating the control function (penalty, no penalty, warning). Regulation is quantified through a set of calculations and constraints.

Figure 13 represents the Trust model. Each PO (through its various roles played in a VO) can have or not functions to control. The function criterion is represented by a class formed by the attributes: name, description, agreement, achievement and execution. The regulation function is characterized in terms of type, description, visibility, frequency and penalization. Figure 14 proposes an instance of the PO "Stockbreeder" and its function "Cattle weight control and evaluation". This function is controlled by a material asset ("weight standard per cattle type") in the top right screen. The bottom right screen shows 
the Trust's graphic representation. The functions to be controlled are represented by rectangles with rounded corners attached to legends describing them ("Classification" and "Meat").

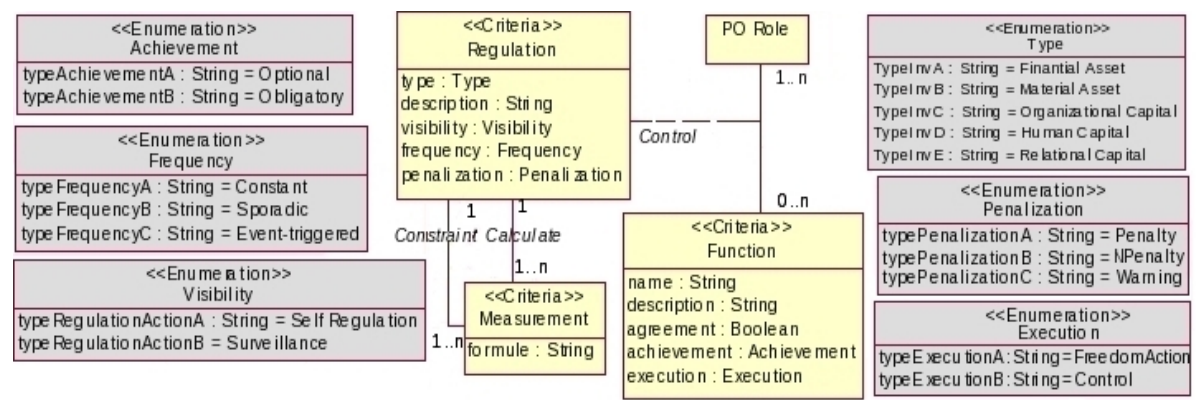

Fig. 13. Trust model

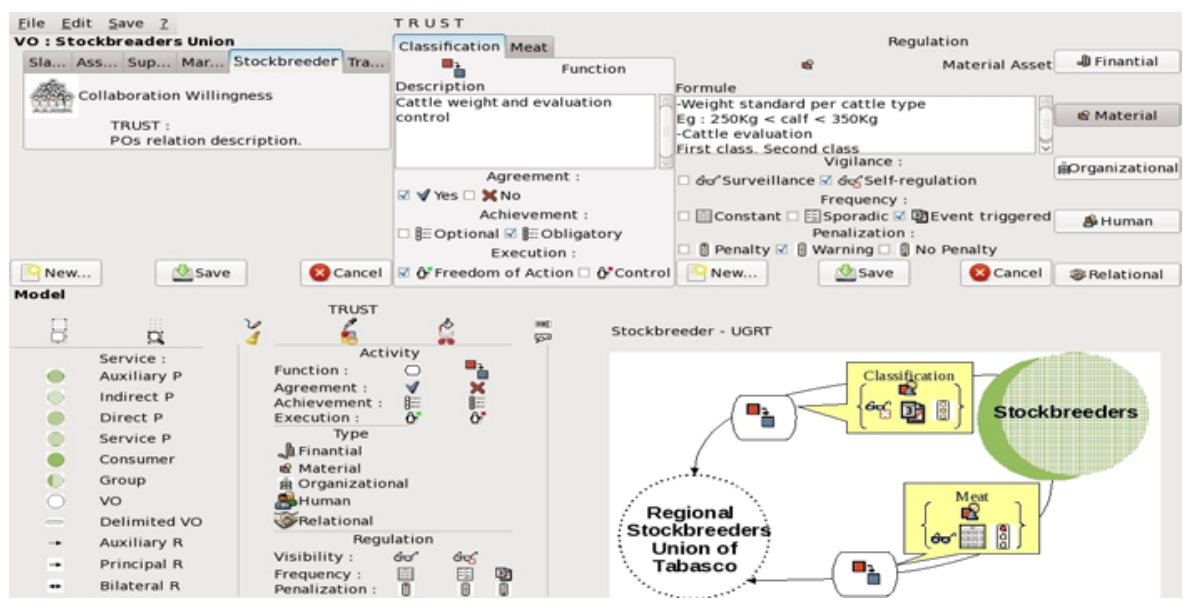

Fig. 14. Trust instance

\section{Conclusion and Perspectives}

This article proposes a collection of criteria to characterize at an intentional level the various participants' organizations within the VO and their interrelationships. These criteria, resulting in particular from work in the management field, are gathered in five aspects. The first two aspects, Identification and Collaboration Willingness are formalized in UML models. The instances come from our case study the Regional Stockbreeders Union of Tabasco in Mexico. The three other aspects (Common Objective, Alliance Formalization and Integration Formalization), are not described in this article because space constraints. 
In addition to this formalization, a modelling prototype tool is under development that will allow the automatic generation of simple graphic models from captured instances. These models are the base for identifying and modelling the requirements that the VO's IS should conform on the basis of the PO's IS. Whith the aid of this prototype the next step is to test the $360^{\circ}$ vision VO model in other service sectors (health, education ...) and to validate it with each PO and the various actors. Moreover, further analysis has to be done to test the model with other types of VO (short term alliance for a Dynamic VO for example) and to refine the proposition at the intra et extra levels.

\section{References}

1. Tripathy, A., Eppinger, S.D.: A system architecture approach to global product development. Sloan School of Management Working Paper (4645-07) (March 2007)

2. White, A., Daniel, M.: Electronique marketplace to marketplace alliances: Emerging trends and strategic rationales. In: ACM Second International Conference on Entertainment Computing, Pittsburgh PA, May 3-10 (2003)

3. Booch, B., Rumbaugh, J., Jacobsen, I.: The Unified Modeling Language User Guide. Addison-Wesley, Reading (1999)

4. Travica, B.: The design of the virtual organisation.: a research model. In: Associations of Information Systems Conference, Indianapolis, August 1997, pp. 15-17 (1997)

5. Freeman, L.C.: Visualizing social networks. Journal of Social Structure 1 (2000)

6. Handy, C.: Trust and the virtual organization: How do you manage people whom you do not see? (May/June 1995)

7. Kasper-Fuehrer, E.C., Ashkanasy, N.M.: Communicating trustworthiness and building trust in interorganizational virtual organizations. Journal of Management (May 1, 2001)

8. Rolland, C., Prakash, N.: Bridging the gap between organisational needs and erp functionality. Requirements Engineering Journal 5, 180-193 (2000)

9. Saunders, C., Wu, Y., Li, Y., Weisfeld, S.: Interorganizational trust in b2b relationships. In: Sixth International Conference on Electronic Commerce. ACM, New York (2004)

10. Power, D.: Supply chain management integration and implementation: a literature review. Supply Chain Management 10(4), 252-263 (2005)

11. den Hengst, M., Sol, G.: The impact of information and communication technology on interorganizational coordination: Guidelines from theory. Special Series on Information Exchange in Electronic Markets 4(3) (2001)

12. Porter, M.E.: Strategy and the internet. Harvard Business Review, 62-78 (March 2001)

13. Barki, H., Pinsonneault, A.: A model of organizational integration, implementation effort, and performance. Institute for Operations Research and the Management Sciences 16(2), 165-179 (2005)

14. Bouarfa, H., Abed, M.: Acquisition of tacit knowledge in virtual organizations. In: CIMCA/IAWTIC, pp. 383-388 (2005)

15. Davidow, Z.H., Malone, M.S.: The virtual corporation: Structuring and revitalizing the corporation for the 21stcentury. T. Harper Business, NY (1992)

16. Fenneteau, H., Naro, G.: Controle et confiance dans l'entreprise virtuelle, illustrations logistiques. IBM Systems Journal, 203-219 (May-June 2005) 
17. Grönniger, H., Krahn, H., Rumpe, B., Schindler, M., Völkel, S.: Text-based modeling. In: Proceedings of the 4th International Workshop on Software Language Engineering (ateM 2007), Nashville, TN, USA (2007)

18. Burn, J., Marshall, P., Wild, M.: Managing knowledge for strategic advantage in the virtual organisation. In: Proceedings of the ACM SIGPR Conference on Computer Personnel Research, New Orleans, USA, April 8-10, pp. 19-26 (1999)

19. Gordjin, J.: Value-based Requirements Engineering: Exploring Innovative eCommerce Ideas. PhD thesis, Vrije Universiteit Amsterdam (2002)

20. Parung, J., Bititci, U.S.: A conceptual metric for managing collaborative networks. Journal of Modellling in Management 1(2), 116-136 (2006)

21. Yu, E.S.K.: Towards modeling and reasoning support from early-phase requirements engineering. In: IEEE 3rd International Symposium on Requirements Engineering, Annapolis MD, January 5-8, vol. 235, pp. 226-235 (1997)

22. Goldman, S.L., Nagel, R.N., Preiss, K.: Agile competitors and virtual organizations: Strategies for enriching the customer. Van Nostrand Reinhold (1995)

23. Camarinha-Matos, L.M., Afsarmanesh, H.: A roadmap for strategic research on virtual organizations. In: Proceedings of IFIP Working Conference on Virtual Enterprises - PRO-VE 2003, Lugano, Switzerland, pp. 33-46 (2003)

24. Brandenbuger, A.M., Nalebuff, B.J.: The right game: use game theory to shape strategy. Harvard Business Review, 57-71 (July-August 1995)

25. Ibrahim, M., Ribbers, P.: Trust, dependence and global interorganizational systems. In: Proceedings of the 39th Hawaii International Conference on System Sciences. IEEE, Los Alamitos (2006)

26. Kanter, R.M.: Collaborative advantage: The art of alliances. Harvard Business Review, 96-108 (July-August 1994)

27. Mazzeschi, M.: The virtual organisation. In: 7th International Conference on Concurrent Enterprising. Bremen, vol. 24(11), June 27-29, pp. 331-335 (2001)

28. Petre, M.: Why looking isn't always seeing: Readership skills and graphical programming. Communications of the ACM 38(6) (June 1995)

29. Roseman, M., Greenberg, S.: Groupkit: A groupware toolkit for building real-time. Conferencing applications. In: CSCW 1992 (1992)

30. Maiden, N.A.M.: Crews-savre: Scenarios for acquiring and validating requirements. Journal for Automate Software Engineering 5(4), 419-446 (1998)

31. Marshal, P., McKay, J., Burn, J.: The tree s's of virtual organisations: Structure, strategy and success factors. School of Management Information Systems, Edith Cowan University (1999)

32. Robinson, P., Karabulut, Y., Haller, J.: Dynamic virtual organization management service oriented enterprise applications. In: IEEE International Conference on Collaborative Computing: Networking, Applications and Worksharing (2005)

33. ECOLEAD Project. Characterization of key components, features, and operating principles of the virtual breeding environment. Afsarmanesh UvA, H. (ed.) D21.1:8 (March 2005)

34. Albert, R., Barabasi, A.L.: Statistical mechanics of complex networks. Reviews of Modern Physics 74 (January 30, 2002)

35. Shani, R., Grant, R.M., Krishnan, R., Thompson, E.: Advanced manufacturing systems and organizational choice: Sociotechnical system approach. California Management Review 34(4), 91-111 (Summer 1992)

36. Sousa, R., Putnik, G.: A formal theory of bm virtual enterprises structures. In: IFIP International Federation for Information Processing, vol. 159 (2005)

37. Basole, R.C., Rouse, W.B.: Complexity of service value networks: Conceptualisation and empirical investigation. IBM Systems Journal 47(1), 53-70 (2008) 
38. Cheng, S., Xu, X., Wang, G., Li, Q.: An agile method of modeling business process simulation for virtual enterprises. In: IEEE International Conference on e-Business Engineering (2005)

39. Iacono, C.S., Weisband, S.: Developing trust in virtual teams. In: Proceedings of the Thirtieth Hawaii International Conference on System Sciences, vol. 2, pp. 412 420. IEEE, Los Alamitos (1997)

40. Nurcan, S., Barrios, J., Rolland, C.: Une methode pour la definition de l'impact organisationnel du changement. In: XXème Congrès INFORSID, Nantes, June 4-7 (2002)

41. Levitt, T.: Production line-approach to service. Harvard Business Review 50(5), 41-52 (1972)

42. Salvador, T., Scholtz, J., Larson, J.: The denver model for groupware design 5yeeeeee haaaaaa! Conferencing applications. In: CSCW 1992, CHI 1995, Denver Colorado, vol. 28(1), pp. 52-58 (1996)

43. Brandes, U., Raab, J., Wagner, D.: Exploratory network visualization: Simultaneous display of actor status and connection. Journal of Social Structure 2(4) (October 19, 2001)

44. Sambamurthy, V., Bharadwaj, A., Grover, V.: Shaping agility through digital options: Reconceptualizing the role of information technology in contemporary firms. MIS Quarterly 2(27), 237-263 (2003)

45. Tatikonda, M.V., MontoyaWeiss, M.M.: Integrating operations and marketing perspectives of product innovation: The influence of organizational process factors and capabilities on development performance. Management Science INFORMS 47(1), 151-172 (2001)

46. van Lamsweerde, A., Darimont, R., Letier, E.: Managing conflicts in goal-driven requirements engineering. IEEE Transactions on Software Engineering 24(11), 908 926 (1998)

47. Barnett, W., Presley, A., Johnson, M., Liles, D.H.: An architecture for the virtual enterprise. In: IEEE International Conference on Systems, Man, and Cybernetics, Humans, Information and Technology, October 1994, vol. 1(2-5), pp. 506-511 (1994)

48. Malone, T.W.: What is coordination theory? In: National Science Foundation Coordination Theory Workshop (February 19, 1988)

49. Laurillau, Y.: Conception et realisation logicielles pour les collecticiels centrees sur l'activite de groupe: le modele et la plate-forme Clover. PhD thesis, Universite Joseph-Fourier Grenoble I (2002)

50. Yang, Z., Zhang, J.B., Low, C.P.: Towards dynamic integration on collaborative virtual enterprise using semantic web services. In: IEEE International Conference on Industrial Informatics (2006) 